\title{
Anhang F: Präsidenten und Sekretäre
}

\section{Januar 1790}

Präsident: Jean Jacques François Théodose Barbier de Tinan

Vizepräsident: Jean Pierre Charles Mayno

Sekretäre: Antoine Genthon, François Laurent Xavier Levrault

\section{Januar $\mathbf{1 7 9 0}$}

Sekretäre: Augustin Médard Lachausse, François Thomas Galbaud dit Dufort (l'ainé), François Jacques Antoine Mathieu (cadet), Thomassin

\section{März 1790}

Präsident: Richard François Philippe Brunck de Freundeck

Sekretäre: François Thomas Galbaud dit Dufort (l'ainé), Antoine Genthon

\section{April 1790}

Präsident: César Galbaud dit Dufort (cadet)

Sekretär: François Jacques Antoine Mathieu cadet

${ }^{1}$ Die vorliegende Aufstellung entstand auf der Grundlage der drei Protokollbände des Straßburger Jakobinerclubs; sie gibt die Daten und Ergebnisse der Wahlen zum bureau im engeren Sinn, d.h. die Wahlen zu den Präsidenten und Sekretären, wieder. Andere Amtsinhaber, wie die Schatzmeister und die Mitglieder der verschiedenen Komitees, wurden nicht berücksichtigt. Nach der Verabschiedung des ersten nicht-provisorischen Reglements im März 1790 wurden zunächst ein Präsident und zwei Sekretäre gewählt. Nachdem sich im Sommer 1790 die zweisprachige Sitzungspraxis durchgesetzt hatte, gab es einen (frankophonen) Präsidenten, einen (germanophonen) Vizepräsidenten, sowie zwei französisch- und zwei deutschsprachige Sekretäre; letztere werden in der vorliegenden Liste, in Anlehnung an die in den Quellen verwandten Begriffe als „deutsche" und "französische" Sekretäre bezeichnet. Grundsätzlich gilt, daß Präsidenten für einen Monat im Amt waren, Sekretäre für zwei Monate; bei jeder Wahl wurde jedoch nur je einer, ab dem Juli 1790 je zwei Sekretäre abgelöst, so daß im Regelfall ein erfahrener mit einem neuen Sekretär arbeitete. Andere Quellen als die Protokollbände, vor allem Unterschriften der Funktionsträger auf Briefen und Veröffentlichungen des Clubs, werden in der vorliegenden Aufstellung nur berücksichtigt, wenn sie zur - häufig schwierigen - Identifizierung einer Person (vor allem bei Mitgliedern mit gleichem Nachnamen) dienen. Da die Bezeichnungen président, bzw. secrétaire auf Briefen und Veröffentlichungen auch einen Vorsitzenden, bzw. Sekretär des Korrespondenzkomitees oder eines anderen Komitees bezeichnen können, sind Unterschriften zur Ausweitung der Liste unbrauchbar. Ein oberflächlicher Blick auf die vorhandenen unterschriebenen Schriftstücke legt jedoch die Annahme nahe, daß Sekretäre weitaus häufiger als aus der vorliegenden Liste hervorgeht zwischen den periodischen Wahltagen ausgewechselt wurden. In der Liste sind die Namen von Personen durch Kursive hervorgehoben, die zwar gewählt wurden, ihr Amt aber nicht antraten oder die nicht identifzierbar sind; dieser Personenkreis wurde bei den in Kapitel II.2 dargestellten Rechnungen nicht berücksichtigt. 
11. Mai 1790

Präsident: Jacques Pierre Simon Brunck de Freundeck

Sekretär: Gaspard Noisette (fils)

\section{Juni 1790}

Präsident: François Thomas Galbaud dit Dufort (l'ainé), Sekretäre: Marie Frédéric Henri Christiani (fils) (lehnt ab); 9. Juni 1790: Amédée Boissière

\section{Juli 1790}

Präsident: Amédée Boissière

Vizepräsident: Friedrich Rudolph Saltzmann

Französische Sekretäre: Joseph Perruquet de Montrichard, Jean François Ehrmann

Deutsche Sekretäre: Andreas Meyer (fils), Jean Philippe Reinbold

\section{August 1790}

Präsident: François Laurent Xavier Levrault

Vizepräsident: Jean Philippe Reinbold

Französischer Sekretär: Pierre Alexis Gloutier

Deutscher Sekretär: Andreas Ulrich

\section{September 1790}

Präsident: Jacques Revel (lehnt ab), 17. September 1790: Friedrich Rudolph Saltzmann

Vizepräsident: Jean Schweighäuser

Französischer Sekretär: André Briche

Deutscher Sekretär: Jean Frédéric Simon; 17. September 1790: Jean François Ehrmann

\section{Oktober 1790}

Präsident: Jean Jacques François Théodose Barbier de Tinan

Vizepräsident: Jean Frédéric Wehrlen

Französischer Sekretär: Alexandre d'Hauterive

Deutscher Sekretär: Jacques Louis Albert

12. November 1790

Präsident: Jean Michel Mathieu (l'ainé)

Vizepräsident: Weilher

Französische Sekretäre: Jean Gaspard Julien Godailh; Antoine Genthon

Deutscher Sekretär: Jean André Kamm 


\section{Dezember 1790}

Präsident: Pierre Alexis Gloutier

Vizepräsident: Mathias Engel

Französischer Sekretär: Louis François Antoine Arbogast

Deutscher Sekretär: Jean Valentinpère Hirschel

\section{Januar 1791}

Präsident: Jean Gaspard Julien Godailh

Vizepräsident: Ehrmann

Französischer Sekretär: Chrétien Valentin Meniolle

Deutscher Sekretär: Louis Schmutz

\section{Februar 1791}

Präsident: Augustin Médard Lachausse

Vizepräsident: Jean Georges Schertz

Französischer Sekretär: Gabriel Bonnard

Deutscher Sekretär: Louis François Antoine Arbogast

18. März 1791

Präsident: Jean Antoine Louis

Vizepräsident: Christoph Willhelm Koch

Französischer Sekretär: Claude Champy

Deutscher Sekretär: Louis Albert (tritt zurück); 2. April 1791: Jean Georges Frühinsholz

\section{April 1791}

Präsident: Michel Thomassin

Vizepräsident: Jérémie Jacques Oberlin

Französischer Sekretär: Alexandre d'Honnières,

Deutscher Sekretär: Jean André Kamm

\section{Mai 1791}

Präsident: Claude Hilaire Laurent

Vizepräsident: Jean Philippe Reinbold

Französischer Sekretär: Alexandre Gérard

Deutscher Sekretär: Jean Philippe Graffenauer; 23. Mai 1791: Jean Chrétien Holtzapfel (cadet) 


\section{Juni 1791}

Präsident: Gaspard Noisette (fils)

Vizepräsident: Jean René Kugler (père)

Französischer Sekretär: Charles P. Perrigny

Deutscher Sekretär: Jacques Dominique Muller

\section{Juli 1791}

Präsident: Charles Sicard

Vizepräsident: Philippe Jacques Engel

Französischer Sekretär: François Xavier Bevalet (tritt zurück); 17. Juli 1791:

Louis Philippe Chayrou

Deutscher Sekretär: Georges Kerner

\section{August 1791}

Präsident: Martin (lehnt ab); 15. August. 1791: Richard François Philippe Brunck de Freundeck

Vizepräsident: Charles Frédéric Vogt

Französischer Sekretär: Gaspard Noisette (tritt zurück); 15. August 1791: Kastner (fils)

Deutscher Sekretär: Roederer (tritt zurück); 15. August 1791: Jean Daniel Stoeber

\section{September 1791}

Präsident: Gabriel Bonnard (le jeune)

Vizepräsident: Frédéric Charles Greuhm (père)

Französischer Sekretär: Camille Louis Laurent Barbier (tritt zurück); 21.

September 1791: Nau Rochefort

Deutscher Sekretär: Frédéric Scherer (fils)

\section{Oktober 1791}

Präsident: Jean Antoine Louis

Vizepräsident: Jean Léonard Froereisen

Französische Sekretäre: Jean François Garnier, Charles P. Perrigny

Deutsche Sekretäre: Carl Clauer, Christoph Friedrich Cotta

\section{November 1791}

Präsident: François Borelle, dit Alexandre

Vizepräsident: Jean Christophe Kienlin (ainé)

Französischer Sekretär: Antoine Claude Dièche

Deutscher Sekretär: Samuel Daniel Stamm (fils) 
12. Dezember 1791

Präsident: Gaspard Noisette (fils)

Vizepräsident: Jacques Dominique Muller

Französischer Sekretär: Chrétien Valentin Meniolle (fils)

Deutscher Sekretär: Holtzapfel

13. Januar 1792

Präsident: Charles Laveaux

Vizepräsident: Eulogius Schneider

Französischer Sekretär: François Grandval

Deutscher Sekretär: Henschel (lehnt ab); Anton Joseph Dorsch

\section{Februar 1792}

Präsident: Philibert Simond

Vizepräsident: Jean Philippe Graffenauer

Französische Sekretäre: Antoine Genthon, Antoine Téterel

Deutsche Sekretäre: Jean Hess, Charles Taffin

\section{März 1792}

Präsident: Alexandre d'Hauterive

Vizepräsident: Jean Christain Ginzrot (fils)

Französischer Sekretär: Pierre François Monet

Deutscher Sekretär: Anton Joseph Dorsch

\section{April 1792}

Präsident: Antoine Téterel

Vizepräsident: Anton Joseph Dorsch

Französischer Sekretär: Thiriet

Deutsche Sekretäre: Rubsamen, Charles Taffin

\section{Juli 1792}

Präsident: Pierre François Monet

Vizepräsident: Edelmann (lehnt ab); Jean Jung

Französischer Sekretär: Adrien Boy

Deutscher Sekretär: Charles Taffin

\section{August 1792}

Präsident: Perrin

Vizepräsident: Jean Philippe Graffenauer

Sekretäre: Mozella, Antoine Lepicq 
1. September 1792

Präsident: Antoine Martial Larivière

Vizepräsident: Jean Jacques Waghette

Französische Sekretäre: Guyenet, Jean François Garnier

Deutsche Sekretäre: Jean Thiebault Hetzel, Bernheimer

1. November 1792

Präsident: Coustard de Saint-Lô

Vizepräsident: Geoffroi Louis Edelmann

Französische Sekretäre: Alexandre de Beauharnais, François Chaudon

Deutsche Sekretäre: Jean Frédéric Kiechel, Jacques Abraham Martin

1. Dezember 1792

Präsident: Michel Rivage

Vizepräsident: Jacques Abraham Martin

Französische Sekretäre: Klein (fils) Armand Maizière

Deutsche Sekretäre: Frédéric Korn, Jean Gambs

1. Januar 1793

Präsident: Armand Maizière

Vizepräsident: Jean Goeffroi Fischer

Französische Sekretäre: Marx-Wolff

Deutsche Sekretäre: Philippe Jacques Engel, Jean Frédéric Kiechel 
Analyse der Präsidenten und Sekretäre

Berufsgruppen ${ }^{2}$

1. Halbjahr 1790

$\begin{array}{cccccccc}\text { Amtsinh./insg. } & B 1 & B 2 & B 3 & M & H & K l & \text { Unbek. } \\ 12 & 1 & - & - & 1 & - & - & 10 \\ 23 & - & 5 & 4 & - & - & 1 & 13 \\ 25 & 1 & 3 & 4 & 1 & - & 2 & 14 \\ 25 & 1 & 5 & 1 & 5 & - & 2 & 11 \\ 14 & - & 3 & 1 & 1 & - & 4 & 5 \\ 24 & - & 4 & 5 & 4 & 3 & 1 & 7\end{array}$

Konfession

Amtsinh./insg. Protestanten Katholiken Unbekannt

1. Halbjahr 1790

2. Halbjahr 1790

12

23

25

1. Halbjahr 1791

2. Halbjahr 1791

1. Halbjahr 1792

2. Halbjahr 1792

Geburtsorte $^{3}$

24
12

10

7

2

6
8

$7 \quad 4$

$10 \quad 5$

$\begin{array}{ll}10 & 8\end{array}$

$7 \quad 5$

7
4 
